\title{
Prospectiva de la investigación socioeducativa en el Perú
}

Jaime RÍOS BURGA

RESUMEN

El presente artículo busca ubicar prospectivamente en el marco sistémico global del cambio de las sociedades las situaciones y problemáticas centrales de la investigación socioeducativa en el Perú de hoy. Destacar algunas de las líneas centrales de investigación y los nuevos retos científicos políticos socioeducativos.

Palabras clave:

\section{Prospective of socio-educational research in Peru}

\section{ABSTRACT}

This article seeks to locate prospectively in the global systemic framework of changing societies and situations central issues of socio-educational research in Peru today. Highlight some of the main lines of research and new socio- political scientific challenges.

KEYWORDS: 


\section{La nueva lógica general de la investigación socioeducativa}

V ivimos cada vez más una sociedad de riesgo (Beck, 2002) en fronteras socioeducativas diferenciadas (Caride, 2005) de comunicación (Castells, 2009). La nueva urbanización y desruralización, la nueva dinámica del poder, el modo de desarrollo infocomunicacional y el cambio transcultural en las sociedades, ubican al nivel primario, secundario y superior en una desigual situación socioeducativa. Se constata que la vida educativa sufre una nueva diferenciación estructural al igual que los logros de aprendizaje, cobertura y matrícula. Los problemas para la conclusión de los estudios en los espacios urbanos rurales también se diferencian en términos sociales e interculturales bilingües unido al abandono escolar, el embarazo adolescente, la desigualdad de oportunidades, la formación y calificación docente. Un desarrollo desigual en la calidad educativa de los egresados y la falta de investigación en la mayor parte de la educación superior.

En este contexto la nueva lógica general de la investigación socioeducativa se ubica y construye hoy como producto del cambio societal global. Las situaciones y problemáticas de investigación surgen como "hechos socio educativos" globales en sus causas, motivaciones, sentidos de la acción y estructuración social en todo su significado social buscando cada vez más comprender, explicar y resolver sus situaciones y problemáticas a partir de modelos teóricos y aplicados cada vez más complejos y multidisciplinarios (Leopardi, 2010).

La nueva investigación socioeducativa como proceso metódico se integra así cada vez más a un paradigma multiparadigmático, múltiple y multidimensional de la ciencia de manera única y diversa. Se integra con los otros campos de la propia sociología y de las otras ciencias en un diálogo pluriparadigmático, plurimetodológico y multidisciplinar. Un nuevo marco metateórico, teórico y temático de investigación de las problemáticas socioeducativas en el permanente cambio societal global.

En nuestra peculiaridad sociohistórica tenemos el desafío de superar, por un lado, las tensiones generadas por las tentativas de imposición de un pensamiento único utilitarista sobre las áreas de colonización y, por otro, el reconocimiento del fracaso de esta ambición totalitaria eurocentrista (Martins, 2012). En otras palabras, en la universalidad del cambio construir conocimientos pertinentes en la dinámica sistémica del cambio del mundo de las organizaciones caracterizadas hoy por la aceleración de los procesos de despersonalización, deslocalización y destemporización de las actividades organizacionales superando así los modelos teóricos que no consideren a la comunicación como su centro (Arnold, 2014).

Un proceso no lineal ni único sino de complejidad donde evalúa, integra y diferencia, teoriza y aplica, cada uno de los paradigmas de investigación en su espe- 
cificidad, unidad y complejidad: Positivista (Racionalista, cuantitativo), socio-crítico, descriptivo, explicativo, predictivo y experimental (Farnos, 2010) en sus cinco fases de investigación: problema, diseño, recolección de datos, análisis de datos, validez de datos, informe de la investigación (Ruiz, 1996). Intercambios de experiencias investigativas que cada vez más en el diálogo vinculan a los investigadores y la investigación concreta con los propios actores de la vida socioeducativa. Como bien destaca Edgar Morin todo en el diálogo tetralógico del orden y el desorden entre física-biologíaantropología-sociología como complejidad de la complejidad del antimétodo hacia el método (Morin, 1999).

Vemos así desarrollarse nuevos modelos de investigación bajo enfoques comprensivo, hermenéutico, cualitativo, complejo, sistémico, constructivista, dialéctico e histórico crítico, ubicando a los nuevos actores socioeducativos como centro del análisis en un enfoque inter, pluri y transdisciplinario combinando creativamente sus marcos teóricos en una hibridez conceptual y metodológica (Torres, 2004) acorde con las problemáticas concretas de investigación. Aquí se hace fundamental potenciar desde la universidad la reflexión teórica, epistemológica, la investigación-acción con programas de intervención socioeducativos concretos (March y Orte, 2015) al servicio del Perú como sociedad política del conocimiento.

\section{Nuevas situaciones y problemáticas}

Vemos surgir así nuevas situaciones y problemáticas socioeducativas en un contexto donde aún todavía falta resolver la universalización principalmente de la educación primaria. Como anota el informe global de seguimiento de la Unesco "Educación para Todos (EPT) 2000 - 2015" el Perú alcanzó cuatro de los seis objetivos educativos acordados en el marco del Foro Mundial de Dakar (Senegal). Según el documento, el Perú al 2015 alcanza las metas de primera infancia, universalización de la primaria, paridad de género en los colegios, y cumplirá con la reducción del analfabetismo.

Al respecto el especialista Idel Vexler, destaca que si bien es cierto el Perú está avanzando en el logro de aprendizaje a nivel primario, lo cual nos permite ubicarnos en casi todos los casos por encima del promedio de los países de Latinoamérica y el Caribe, aún no se ha cumplido con los objetivos sobre calidad educativa, y a la atención para que los jóvenes y adultos logren competencias de aprendizaje significativas. Destaca: "Esto es preocupante y debe llevarnos a una gran reflexión para tomar medidas de mejoramiento institucional, administrativo y pedagógico de nuestro sistema educativo nacional, priorizando los ámbitos rurales e interculturales bilingües" (Vexler, 2015). 
En cuanto a los resultados de la región sobre la primera infancia, el informe indica que un poco más de la mitad de los países de la región, entre ellos Chile, Ecuador, México y Perú alcanza el $80 \%$ o más de la matrícula en la educación inicial (3 a 5 años). Sobre la universalización de la primaria, señala que un poco más del $50 \%$ de los países, entre ellos Perú, ha logrado la universalización de la educación primaria. En torno al analfabetismo, solamente Bolivia, Perú y Surinam alcanzarán la meta fijada en alfabetización y otros cinco más se acercan a su obtención. Sobre paridad de género, el $60 \%$ de los países de la región ha logrado la paridad entre mujeres y varones en la primaria, pero en la secundaria ese porcentaje se encuentra tan sólo en un $20 \%$. El Perú en los centros de primaria y secundaria logra esta meta.

Situación socioeducativa que se hace más diversa si analizamos la diferenciación en la educación, principalmente superior. En este marco podemos ubicar nuevas áreas de problemáticas socioeducativas de investigación como:

- Educación, teorías, metodologías y epistemologías socioeducativas.

- Sociología del currículo.

- El impacto de la globalización capitalista en la vida socioeducativa.

- La relación entre educación y actores sociales diversos en sus contextos específicos urbanos rurales.

- Estratificación social y actores socioeducativos: individuo, familia, grupos sociales.

- Socialización educativa: Escuela, universidad, familia, medios.

- Educación y mercado de trabajo.

- Educación y empresa global.

- Educación ambiental y desarrollo sustentable.

- Educación y nueva dependencia global.

- Educación, desigualdad, pobreza, género y exclusión social.

- Educación y sociedad global de la información y comunicación.

- Educación, territorios y descentralización.

- Educación, ciencia, tecnología e innovación.

- Educación e institucionalidad nacional global.

- Educación, acciones colectivas y movimientos sociales.

- Violencia, comunidad y sociedad educativa.

- Sistemas educativos globales.

- Educación, docentes y autoridad.

- Educación y abandono escolar.

- Educación y marginalidad. 
- Educación, Estado y políticas educativas: estrategias, metas y medidas de política pública: presupuesto, calidad, equidad, mercado, gestión, integración, infraestructuras, etc.

- Educación y modelos socioeducativos urbano rurales.

- Educación, consumos culturales y medios de comunicación.

- Educación, interculturalidad y transculturalidad.

- Educación, imaginarios y memorias.

- Educación, cuerpos y emociones.

- Educación, niñez, juventud y envejecimiento.

- Educación, corrupción y violencia.

- Educación, ocio y deporte.

- Educación y vida cotidiana.

Podemos formular aquí de acuerdo al interés y preocupación científica social socioeducativa diversas preguntas de investigación como por ejemplo: ¿Cuáles son los factores socioeducativos que llevan a producir y reproducir la dependencia científica tecnológica en los peruanos de manera recurrente hasta hoy? ¿Cómo la globalización del capitalismo cognitivo actual afecta a los actores socioeducativos? ¿Cómo la diversidad de los actores socioeducativos en el Perú afectan los diferentes hábitos socioeducativos? ¿El bajo nivel de rendimiento escolar en el país se vincula a una sociedad predominantemente oral-audiovisual más que escribal? ¿Cuáles son las causas sociopolíticas de la falta de una institucionalidad socioeducativa nacional global en el Perú de hoy? ¿De qué manera la nueva familia monoparental afecta la socialización y vida socioeducativa de los alumnos en el aula? ¿Cuáles son los problemas socioeducativos concretos presentes en sus espacios y actores micro socioeducativos? ¿Cuáles son los proyectos de intervención socioeducativa urbano rurales que gesten un verdadero desarrollo integral nacional global?

\section{La investigación socieducativa}

La investigación socioeducativa diseña sus propias estrategias acorde con el carácter de sus preguntas y objetivos de investigación. Utiliza con imaginación y creatividad científica la observación, el diálogo, la experimentación, la explicación y/o comprensión, en la búsqueda de conocimientos demostrables, comparables, medibles y/o cualitativos relevantes. La elaboración de modelos, teorías, proyectos y programas de intervención buscando la solución específica de las problemáticas socioeducativas con una visión de futuro. 
Podemos desarrollar investigaciones socioeducativas cuantitativas o/y cualitativas. Dar respuesta a por ejemplo a la siguiente pregunta central: ¿Cómo la diversidad de los actores socioeducativos en el Perú afecta los diferentes hábitos socioculturales de educación y aprendizaje? Pregunta que puede conducirnos a formular la siguiente hipótesis: la actual diversidad de hábitos de los actores socioeducativos en el Perú afecta de manera diferenciada la vida educativa y el aprendizaje en sus diferentes niveles. Hipótesis que para su conocimiento y demostración nos plantea la construcción de marcos teóricos y empíricos específicos. Podemos aquí construir un marco teórico constructivista estructural como lo hace Pierre Bourdieu (Bourdieu, 2012, 2010, 2009, 2008, 1970) para sus investigaciones de la educación francesa:

\begin{tabular}{ll}
\hline Dimensiones & Variables e indicadores \\
\hline Diversidad estructural de actores & Capital económico \\
& Capital social \\
& Capital cultural \\
Hábitos socioeducativos & Capital simbólico \\
& Socioeconómico \\
& Familia \\
\hline Aprendizaje & Lengua \\
& Religión \\
& Mentalidad \\
\hline & Escritura \\
Lógica \\
Matemática \\
Emocional \\
Audiovisual \\
Estético \\
\hline
\end{tabular}

Vemos como un diseño de investigación supone asumir un marco o enfoque teórico específico o integrado planteando construir sobre la problemática un sistema de conocimientos, hipótesis, variables e indicadores, con un universo de muestra cuantitativo y/o cualitativo representativo.

Pero, también podemos desarrollar investigaciones desde otros paradigmas como el paradigma cualitativo, fenomenológico, etnográfico, dramatúrgico, sociotecnológico, donde a diferencia del modelo científico clásico de explicación, predicción y control positivista damos importancia al significado de la acción subjetiva, intersubjetiva y simbólica de los actores en sus contextos concretos de reproducción social concretos. Por tanto, prestar atención al carácter de las interacciones sociales con el objetivo práctico de mejorar o crear las mejores condiciones socieducativas. 
En esta perspectiva desarrollar investigaciones desde el paradigma sociocrítico buscando ir más allá del paradigma positivista e interpretativo uniendo la explicación con la comprensión. Enfoque en que la autoreflexión ideológica crítica ocupa un papel central, pues le da una mayor atención a la transformación de las situaciones y problemáticas socioeducativas existentes. Un episteme de la realidad como praxis construyendo teorías, modelos, evaluaciones, políticas, intervenciones, buscando la solución estructural de los mismos. Teoría y práctica científica social que en la unidad de sus diferencias se enrumban más allá de la racionalidad instrumental y técnica. Perspectiva que se preocupa por construir una formación integral de la vida socioeducativa en sus cambios y permanencias, práctica emancipadora, organización democrática, práctica cotidiana e investigación acción participante.

El modelo sistémico en sus enfoques de sistemas abiertos o sistemas cerrados nos da otra perspectiva de paradigma investigación socioeducativo. La diferenciación interna de la problemática educativa es investigada en sus funciones y procesos de segmentación, estratificación y diferenciación funcional. Sistemas como instituciones relevantes acorde con su propio modelo sistémico o la influencia de los modelos sistémicos norteamericano, británico, alemán, francés, etc.; buscando descubrir el entramado de sus recursos organizacionales como discurso autopoiético de decisiones en la investigación. Todo en la búsqueda de la verdad, la interacción profesor-alumno, los desafíos económico-sociales, políticos, jurídicos, en sus entornos buscando mejorar sus decisiones, medios y códigos en sus circuitos de jerarquía y horizontalidad institucional, membresía y roles de profesores e investigadores, programas (misiones, teorías y currículos) y cultura organizacional de los sistemas educativos (Paulus, 2006).

Por otra parte, el modelo y enfoque integrado de acuerdo a la naturaleza de las preguntas y objetivos de investigación nos lleva a enriquecer la investigación socioeducativa. La metodología como la teoría de los métodos guía aquí el uso de los métodos mixtos y las diferentes técnicas cuantitativas y/o cualitativas buscando la comprensión y/o explicación de los fenómenos socioeducativos. Para ello como recomiendan los especialistas de metodología se hace fundamental determinar el nivel de independencia/interacción entre las fases/métodos (cuantitativa-cualitativa), determinar la prioridad del enfoque cuantitativo o cualitativo, determinar el momento de lo cuantitativo o cualitativo (concurrente, secuencial, multifase), y determinar cuándo y cómo mezclar las fases (interpretación, análisis, recogida, diseño).

La heterogeneidad estructural y los nuevos procesos de homogenización socioeducativa en nuestros países producto principalmente del impacto del capitalismo cognitivo global hacen necesaria las investigaciones en diferentes enfoques o miradas, pero cada vez más en un mayor diálogo e integración teórica, institucional y social superando así el desorden y la ineficiencia como destaca Pearse para el caso de la educación 
primaria en América Latina: “El desorden y la ineficacia de la educación primaria en América Latina no son accidentales y pueden ser analizados sociológicamente. En este ensayo se ha centrado la atención en un aspecto particular. De acuerdo con el modelo utilizado, podemos afirmar que existe una deficiencia de poder operativo en el agente (gobierno) y en el sector más vasto del objeto ("indios", campesinos, los"indigentes", las clases populares, etc.), que desvirtúa la instrumentalidad y abre el camino a procesos de modificación y transformación que pueden ser generalizados bajo dos encabezados, a saber "apropiación institucional" y "apropiación de clase". (Pearse, 2006)

La investigación nos permite utilizar creativamente una diversidad de metodologías, métodos, técnicas e instrumentos. Esquemas teóricos y estrategias cualitativas y/o cuantitativas que acorde con las preguntas y objetivos ordenan los conceptos, datos, análisis, síntesis, etc. Aquí, el diseño muestral en sus diferentes tipos se relacionan con el estudio de casos, la observación, el taller participante, el focus group, el muestreo opinático, el muestreo teórico y modélico, buscando la mejor codificación del lenguaje de los actores socioeducativos, la fiabilidad de los elementos de medición y cualificación a partir de los mayores índices de calidad.

La investigación socioeducativa puede también utilizar de acuerdo a cada problemática de manera fecunda las diferentes técnicas cuantitativas (el sondeo, el experimento, el análisis estadístico, etc.) y cualitativas (la observación, la entrevista en profundidad y la lectura de textos). Un esfuerzo investigativo donde los datos obtenidos nos muestren en toda su expresión el mundo de los actores educativos en su objetividad y subjetividad, intersubjetividad y mundo simbólico. Por ejemplo, la investigación cualitativa puede utilizar los siguientes instrumentos:

MODOS DE RECOLECCIÓN DE INFORMACIÓN CUALITATIVA

\begin{tabular}{|l|l|l|l|}
\hline & Observación & Entrevista & Lectura \\
\hline Directa & Contemplación & Conceptos & Comprensión \\
\hline Soterrada & Proyección & Analogías & Analogías \\
\hline
\end{tabular}

FUENTE: Ruiz: 1996.

La contemplación nos permitirá observar directamente las problemáticas socioeducativas desde fuera o como observador participante. La proyección posibilitará recoger información a partir de la explicación que dan los propios actores en su contexto y momento intersubjetivo. La conversación conceptual nos permitirá recoger información utilizando la técnica mayéutica del diálogo objetivo e intersubjetivo de los actores socioeducativos a partir de la entrevista a profundidad. El intercambio metafórico como técnica posibilitará recoger información y dirigir la gestión en la vida cotidiana entre el investigador y los actores. La comprensión ubicará los datos 
proporcionados por los actores como un texto contextualizado para ser analizados desde la perspectiva de la pragmática lingüística o la semiótica. El palimpsesto posibilitará recoger la información e interpretar un texto u testimonio en sus diferentes significados vividos por los actores.

El análisis de los datos se orientará por una metodología que integra de manera creativa los métodos y las técnicas cualitativas y/o cuantitativas. Los métodos cuantitativos analizan las problemáticas fundamentalmente mediante la constatación de frecuencias (parámetros), coincidencias (asociaciones), algoritmos (medias, desviaciones, ratios, coeficientes, etc.), test de asociación, test de correlación, test de regresión, test de diferencia significativa.

Los métodos cualitativos emplearán los instrumentos de la observación, la entrevista, la lectura de textos, la cámara de video, el magnetófono. Los datos obtenidos permitirán descubrir y comprender las problemáticas en sus patrones de estructuración, acción e intereses socioeduactivos y culturales. Aquí, podemos construir acorde con cada situación modelos, tipos ideales, definiciones, biografías, matrices, etc.

La investigación socioeducativa planifica el método y la técnica a utilizar en función de los datos que dispone y el diseño general de la investigación. La abstracción científica y la experimentación adquieren una nueva importancia junto a los métodos de la analogía, formalización, matematización, modelación (Velasquez y Rey, 1999). Procedimientos que empleamos con el objetivo de llegar al conocimiento de verdades generales o particulares (De Hostos, 2006).

Para su validez la metodología combina creativamente la vía inductiva-empírica y la deductiva-teórica construyendo en la validación conceptos, proposiciones, modelos y teorías. La validez metodológica como producto de la dialéctica teoría y práctica.

La epistemología de la ciencia percibe hoy la unidad y diferencia de la realidad socioeduactiva como homogeneidad y heterogeneidad estructural de la acción social de los actores en el cambio global. Aquí el conocimiento de las relaciones sociales es construido sus patrones, procesos y tendencias de estructuración socioeducativa en sus permanencias y cambios sociales. Las condiciones objetivas de los distintos tipos de discurso no se reducen a un modelo estructural a secas sino captan la acción socio histórica de los actores socioeducativos en su contenido relacional esencial y multidimensional. La explicación y comprensión se combinan creativamente vinculando lo macro y/o lo micro en un estilo integrado y diferenciado de investigación.

Un nuevo paradigma investigativo que construye el análisis social de lo real y subjetivo, lo consciente e inconsciente como relaciones sociales totales e históricas sin perder de vista los profundos cambios presentes a niveles de la individuación, socialización, identidades e imaginarios. En otras palabras, un episteme verdaderamente social y sociológico que supera todo psicologismo, formalismo o matematización 


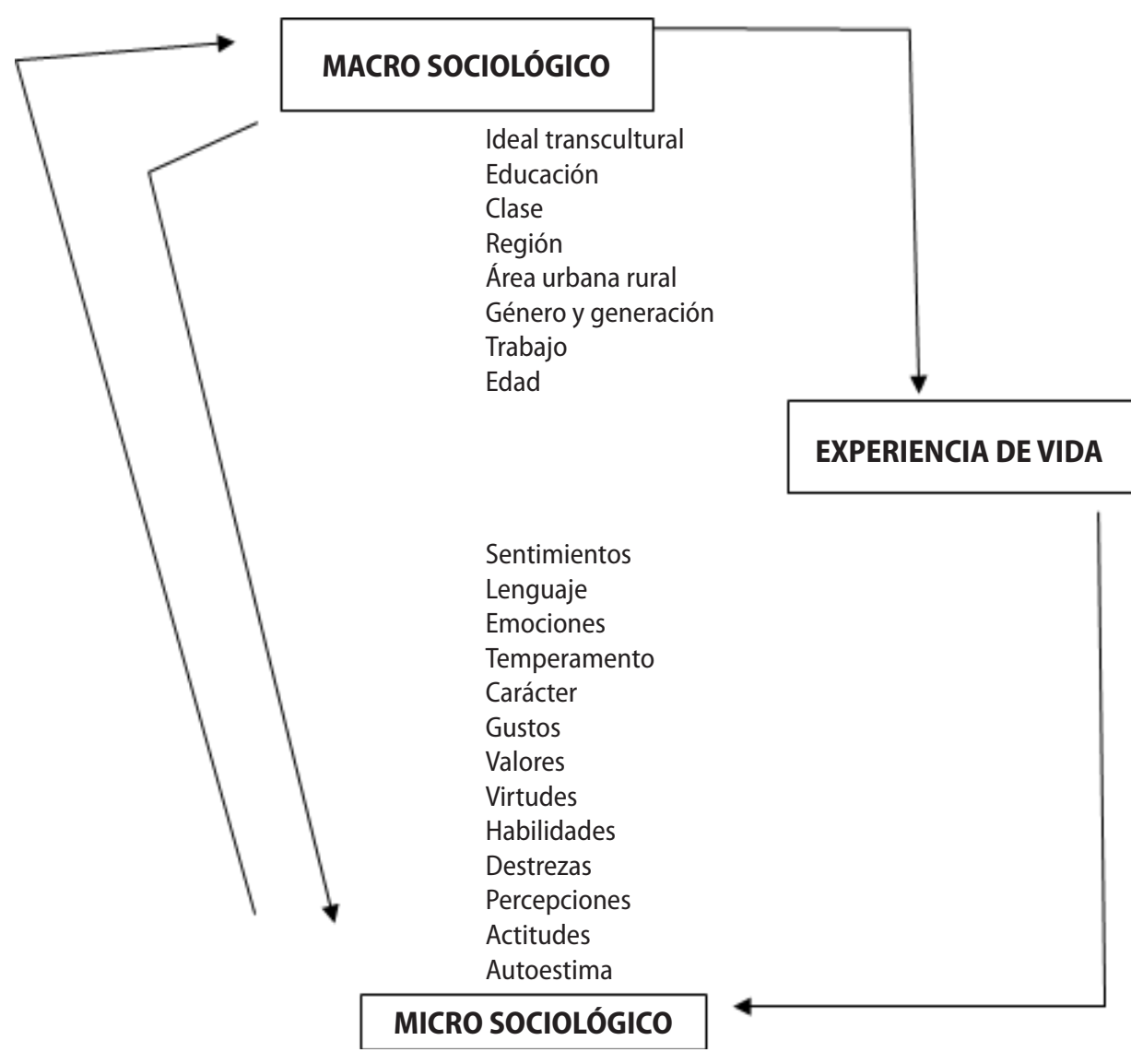

unilateral. Un nuevo esquema de investigación socioeducativa que en sus niveles macro micro tome en consideración los siguientes aspectos:

Una metodología creativa y crítica que capta los nuevos patrones y tendencias de la sociedad del conocimiento e información en sus determinaciones e indeterminaciones, simplicidad y complejidad guiados por un conocimiento racional, sistémico, exacto, verificable y falible integrando desde una filosofía de una cultura de vida el método cientíico a partir de teorías y modelos concretos. Una metodología que sigue el siguiente esquema (ver página siguiente).

Si la metodología nos proporciona la teoría de los métodos y las técnicas de investigación a utilizar acorde con cada tipo de problemática socioeducativa, la epistemología proporciona los criterios de cómo construimos conocimientos. Los métodos de pensamiento y acción nos permiten utilizar los procedimientos más adecuados. Por ejemplo, los métodos de pensamiento posibilitan hacer uso de los conceptos y las proposiciones para construir abstracciones, definiciones, divisiones, 


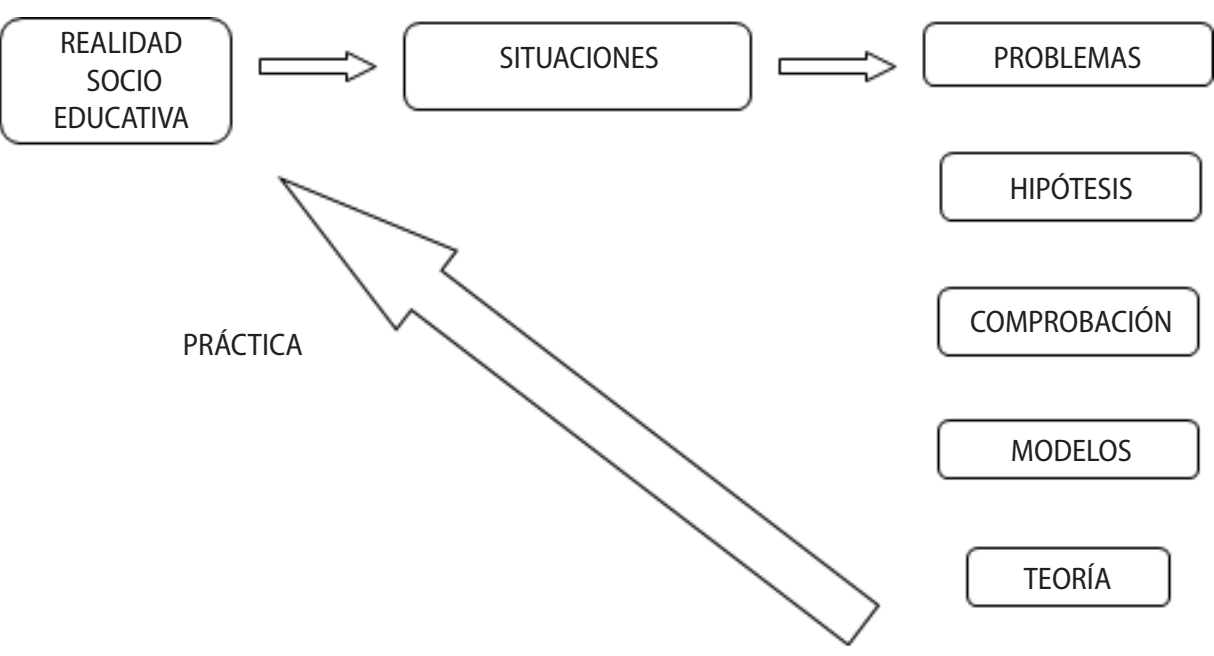

clasificaciones. Los métodos de razonamiento nos posibilitan la deducción, la inducción, el análisis, la síntesis, la comparación, las diferencias, etc. Una epistemología que nos hace conocer los mejores criterios para la construcción de conocimientos relevantes de un mundo socioeducativo cada vez más transcultural. Notamos aquí la transición del modelo epistémico científico clásico de la simplicidad a otros modelos epistemológicos más complejos.

Mientras el modelo epistemológico de la simplicidad se caracterizaba por el uso de método inductivo y el método hipotético inductivo hoy asumimos los modelos complejidad superando la separación del sujeto del objeto de conocimiento y solo descubrir las leyes invariantes en su causalidad última. El modelo de simplicidad por el carácter absoluto de sus enunciados científicos le llevaban ilusoriamente a perder de vista la complejidad del orden y el caos (Prigonini, 1990).

El modelo inductivo parte del conocimiento de casos concretos, utiliza el análisis y la síntesis para hacer generalizaciones sobre la problemática en investigación. El modelo hipotético deductivo desarrolla otra forma de conocer la realidad social. Parte de una hipótesis que se formula a partir de la observación y experimentación de la realidad social. Los métodos inductivo e hipotético deductivo van dando paso a un modelo integrado de conocimiento donde la inducción y la deducción forman parte del conocimiento sistémico en su totalidad, historicidad, relaciones estructurales y funcionales. Un modelo epistemológico que revoluciona los dos modelos anteriores para dar origen a un modelo epistemológico de la complejidad.

El nuevo modelo epistémico de la complejidad como modelo sistémico integra a todos los modelos anteriores. Construye un paradigma múltiple a partir de las problemáticas dando cuenta de los fenómenos socioeducativos. Integra la inducción, deducción, 
análisis, síntesis, experimento, observación, abstracción y concreción en todas las fases de la investigación: Problema, hipótesis, teoría, práctica. El sujeto como investigador dialoga con el sujeto investigado en todas sus manifestaciones. Un sujeto que vive en una sociedad del conocimiento donde la teoría y la práctica se integran en la unidad de sus diferencias organizando la vida socioeducativa con calidad de vida.

Por sus relaciones, totalidad e historicidad el paradigma de la complejidad contiene un conjunto de modelos de explicación, comprensión y descripción en sus estructuras, acción, subjetividad, intersubjetividad y mundo simbólico. Toma como centro de preocupación descubrir los procesos de permanencia, cambio y reproducción socioeducativa. Una visión científica diferente al de la modernidad clásica donde se dividía las disciplinas en campos estancos. Un enfoque integrado que une el trabajo disciplinario, multidisciplinario y transdisciplinario entre sociología, antropología, historia, psicología y los otros campos de la ciencia.

El sentido de simulación cobra fuerza integrando macro-micro, teoría-modelo, las problemáticas en un continuo reajuste y cambio teórico metodológico en sus diferentes situaciones. Un esquema epistemológico que transforma lo humano en todos sus espacios y tiempos socioeducativos y culturales con una visión prospectiva de futuro. Un modelo de integración transcultural donde la sociabilidad y la individualización, transforman los entornos en toda la unidad de sus diferencias socioculturales. En otras palabras, la investigación socioeducativa utilizará creativamente la investigación experimental y no experimental a partir de cuatro modelos de diseño (Bericat, 1998):

a) El modelo simple con sus variantes de complementación, combinación y triangulación. El modelo de complementación puede en su dimensión y carácter distributivo basarse en la encuesta; la estructural en los grupos de discusión; y, la dialéctica en el socioanálisis. El modelo de combinación basarse en el uso de la encuesta estadística y la entrevista, es decir, la cuantitativa y cualitativa entre estos dos instrumentos. El modelo de triangulación basado en el cuestionario con criterio estadístico y la teoría combinando la medición múltiple y contrastación de las hipótesis.

b) El modelo evaluativo puede integrar la entrevista, la observación participante, la encuesta sistémica, los grupos de discusión, etc.

c) El modelo complejo recurre a los diferentes métodos, técnicas e instrumentos acorde con la problemática

d) El modelo de triangulación usa diferentes métodos, técnicas e instrumentos para un mismo objeto de investigación, es decir, una combinación creativa en el tratamiento de todas las fuentes de datos según el espacio, el tiempo y la personas; la triangulación de investigadores como múltiples observadores; la 
aplicación de las diferentes metodologías al interior de un método o intramétodo, métodos o intermétodo; $y$, la integración de los aportes teóricos, éstos condicionan el tipo de diseño acode con la problemática evitando construir o sobreponer la teoría a la realidad.

Las teorías sociológicas integradas nos darán un enfoque de tratamiento de la problemática socioeducativa en sus diferentes dimensiones. Sus aportes nos permitirán describir y explicar los hechos socioeducativos ubicando los problemas socieducativos en el marco de la estructura y acción de sus agrupamientos, integraciones y conflictos. Construir las situaciones socioeducativas típicas de significado de acción de los actores en su cotidianidad.

En este marco podemos plantear diferentes diseños: a. Fuera del sistema escolar con las causas que la originan. Por ejemplo, el impacto de la socialización familiar en el estudiante o la influencia de la clase social al cual pertenece el educando. b. Dentro de la escuela investigar el impacto de socialización en su complejo de relaciones entre profesores y alumnos, la autoridad y los actores educativos. c. Las características del estudiante donde se integren la investigación cuantitativa y cualitativa midiendo y cualificando las características de reproducción socioeducativa.

Podemos también ubicar los tipos de socioaprendizaje acorde con cada tipo de diseño:

TIPOS DE EXPLICACIONES SOCIOLÓGICAS DEL APRENDIZAJE ORÍGENES DE LA CAUSA

\begin{tabular}{|l|l|}
\hline Fuera de la Escuela & Dentro de la Escuela \\
\hline 1. Características mentales & 5. Origen causal \\
\hline 2. Características físicas & 6. Consecuencias físicas \\
\hline 3. Características étnicas y socioeconómicas & 7. Estructura social \\
\hline 4. Condiciones ecológicas & 8. Aspectos tecnológicos de la Escuela \\
\hline
\end{tabular}

Walle:1969: Capítulo I

Podemos así mismo, construir diseños de investigación con enfoques integrados como lo hace por ejemplo Flandes cuando destacó que el estilo de enseñanza producía el mayor incremento en el aprendizaje del estudiante, utilizando un esquema de codificación de interacción de grupos pequeños. Investigación que hoy se ve corroborada por saber que el mejor ambiente socioeducativo favorece un mejor aprendizaje.

Por otra parte, como lo hizo Rosental analizar los efectos de las expectativas del maestro en el desempeño de los alumnos a partir de una investigación experimental. Desarrollar como los hacen James Coleman, Ernest Campbell y un equipo de investiga- 
dores un estudio sobre la igualdad de oportunidades educativas utilizando la técnica de la encuesta. 0 , también como lo hizo Shodbeck, desarrollar estudios de una muestra pequeña de jóvenes de educación considerando los hogares, es decir, la relación de los padres e hijos con el objetivo de conocer cómo se da el proceso socioeducativo del conocimiento para el logro de la mejor formación de los niños y jóvenes.

Aquí se hace fundamental los programas de intervención con impactos medibles en sus resultados. Para ello como destaca Gonzalo Pérez se hace fundamental formar equipos de investigación acción para mejorar el desempeño y desarrollo socioeducativo en el aula y sus entornos socio culturales como también el tiempo libre, la educación de adultos, la educación especializada, la formación laboral en ámbitos como la educación ambiental, la salud, la paz, de los actores/as educativos. Una tarea donde el profesor y el estudiante superan las dificultades educativas, aprendizaje y enseñanza superando los bajos rendimientos en calificaciones, los bajos niveles de logro en las asignaturas, el incumplimiento de deberes y tareas, la inasistencia, la desunión y conflicto entre los estudiantes, la falta de interés de los estudiantes. Por tanto, hacemos explícita la visión, misión y políticas programadas identificando el problema, el diagnóstico de fortalezas, oportunidades, debilidades y amenazas (FODA), el modelo operativo en sus variables, indicadores e índices concretos, la medición de los resultados y la evaluación integral continua (Pérez, 2011).

\section{Los nuevos retos socio educativos globales}

La transformación cognitiva global afecta diferenciadamente las problemáticas socioeducativas en el Perú en nuevas dinámicas de integración, desigualdad y exclusión socioeducativa. Se hace aquí fundamental orientar la investigación con una visión global y cotidiana de la vida.

Una primera línea de trabajo de investigación socioeducativa con impacto práctico es la integración actores y sociedad, actores y Estado con una visión nacional global. La sociología de la educación unida a la sociología política y sociología de las organizaciones contribuirá aquí a las mejores formas de gestión promoviendo la mejor centralización y descentralización socioeducativa en sus diferentes niveles. La integración de la educación superior es una de las tareas estratégicas superando la dependencia educativa global. Una planificación que geste en la interdependencia políticas concretas de verdadero desarrollo socioeducativo desde sus entornos.

Una segunda línea de acción es adecuar la educación al nuevo paradigma o modelo de desarrollo de la sociedad de la información y la comunicación. Aquí los modelos socioeducativos en red de redes y equipos bajo programas específicos de investigación 
cobran importancia. La educación va más allá de la instrucción afirmando la autonomía, la creatividad, la innovación, la libre elección de los actores en sociedad. Es decir, aprender a aprender, aprender haciendo y aprender a ser personas al servicio de la vida y la tierra planetaria (Morin, 1999). Una educación que integre texto e hipertexto en el dominio de los distintos niveles de lo virtual, en torno a la comunicación, la construcción de o real y los mundos posibles (Bruner, 1988, 1986, 1984, 1978, 1972) y dando cabida a los distintos niveles de la representación: la acción, la imagen y la palabra. El desafío de convivir, educar y pensar entre la linealidad textual y la hipertextualidad; entre narrativas analógicas y digitales; entre lo presencial y no presencial. Los intercambios, la interpretación y la producción sostenidos en la palabra, el libro y la pantalla

Una tercera línea de investigación que integra la educación infantil, la media y la superior. Aquí se hace fundamental crear un verdadero sistema integrado desde la diversidad de nuestra unidad como sociedad nacional global.

Una cuarta línea integra la educación para el conocimiento y el trabajo acorde con las necesidades de los entornos socioeconómicos, políticos y culturales cambiantes buscando que las diferentes organizaciones confluyan en una nueva cultura de empresa-trabajo social creando nuevas formas de organización ante el creciente desempleo estructural e individuación individualizada de la vida social. Trabajo y ocio saludable se convierten aquí en dos esferas unidades al servicio de la mejora de la calidad de vida de las poblaciones urbano rurales.

Una quinta línea es repensar la formación docente en una cultura educativa al servicio de la vida colectiva e individual integrando teoría y práctica a partir de las nuevas situaciones y problemáticas.

Una sexta línea es nuestra integración socioeducativa al mundo, América Latina y el Caribe.

La crisis de la modernidad global nos ubica ante una nueva dinámica societal. A puertas de la cuarta revolución industrial pos digital y el bicentenario de nuestra independencia se hace fundamental crear la consciencia y visión que vivimos el desarrollo de una cultura civilizatoria transcultural donde alejados de todo etnicismo y nacionalismo cerrado, construyamos desde nuestras diversidades una sociedad peruana del conocimiento donde la peruanidad universal se integra como humanos a la vida como un todo. La relación humanos-naturaleza-cosmos crea nuevos mecanismos de integración global entre los pueblos, estados y sociedades. He ahí la razón de que los problemas socioeducativos son hoy cada vez más globales en la especificidad de sus soluciones. La racionalidad tecnocientífica socioeducativo encuentra aquí prospectivamente su verdadera ubicación y curso de solución en programas de desarrollo reales que resuelvan los problemas concretos de la vida socioeducativa en todas las dimensiones glocales (Castells, 2004). 


\section{Referencias bibliográficas}

ARNolD, M. (2014). "Imágenes de la complejidad: la organización de las organizaciones” en Arnold, M. Cadenas, H. y Urquiza, A. La organización de las organizaciones sociales Aplicaciones desde perspectivas sistémicas. Santiago: Ril editores.

BECK, U. (2002). La sociedad del riesgo global. Madrid: Siglo XXI Editores.

BERICAT, E. (1998). La integración de los métodos cuantitativo y cualitativo en la investigación social Significado y medida. Barcelona: Editorial Ariel S.A.

BIANCHETTI, R. (2016). Educación y Trabajo: un debate fundamental para reconquistar el valor de lo social. CISEN (Centro de Investigaciones Socioeducativas del Noroeste). CIUNSa. (Consejo

BORDIEU, P. (2012). La distinción. Madrid: Taurus.

BoRDIEU, P. (2012). Intelectuales, política y poder. Madrid: Cegal.

BorDIEU, P. (2010). Capital cultural, escuela y espacio social. Madrid: Siglo XXI Editores.

BORDIEU, P. (2009). Los herederos. Los estudiantes y la cultura. Madrid: Siglo XXI Editores.

BORDIEU, P. (2008). Homo academicus. Madrid: Siglo XXI Editores.

BORDIEU, P. (1070). La reproducción. Elementos para una teoría del sistema de enseñanza. Madrid: Cegal.

BRUNER, J. (1988). Desarrollo cognitivo y educación. Madrid: Ediciones Morata.

BRUNER, J. (1986). Realidad mental y mundos posibles. Barcelona: Gedisa.

BRUNER, J. (1984). El desarrollo de los procesos de representación. En Acción, pensamiento y lenguaje. Madrid: Alianza Editorial.

BRUNER, J. (1978). El proceso mental en el aprendizaje. Madrid: Ed. Narcea.

BRUNER, J.(1972). El proceso de educación. México: Ed. Uteha.

CARIDE, J. (2005). Las fronteras de la pedagogía social. Barcelona: Gedisa.

CASTELLS, M. (2009). Comunicación y poder. Madrid: Alianza Editorial.

CASTELLS, M. (2004). La sociedad de la era de la información. Vol. I, II, III. Madrid: Siglo XXI.

De Hostos, E. (2006). Tratado de sociología. Madrid: CIS-BOE

LEOPARDI, L. (2010). La investigación socio-educativa. Hacia un pensamiento complejo. En revista electrónica diálogos educativos issn 0718-1310 n 19 Venezuela Universidad Pedagógica Experimental Libertador, IPM Venezuela.

MARCH, M. Orte, C. (2015). Perspectivas y retos de la investigación. Balears.En http://www. academia.edu/9285446/Perspectivas_y_retos_de_la_investigaci\%C3\%B3n_para_la_Pedagog\% C3\%ADa_Social. España: Universitat de les Illes Balears.

MARTINS, P. (2012). La decolonialidad de América Latina y la heterotopía de una comunidad de destino solidaria. Buenos Aires: CICCUS-Estudios Sociológicos editora.

MORIN, E. (1999). Los siete saberes necesarios para la educación del futuro. Paris: UNESCO.

MORIN, E. (1999). El método La naturaleza de la naturaleza. Madrid: Ediciones Cátedra S.A.

\section{6/ REVISTA DE SOCOLLOGíA 26}


PeARSE, A. (2006). Problemas Estructurales de Sistemas Educativos en América Latina. IIDS. Ginebra: ONU.

PÉREZ, G. (2011). La intervención socioeducativa. En http://uvprintervencioneducativa. blogspot.pe/2011/09/la-intervencion-socioeducativa-por.html

RuIz, J. (1996). Metodología de la investigación cualitativa. Bilbao: Universidad de Deusto.

PAULUS, N. (2006). Las universidades desde la teoría de los sistemas sociales. En Calidad de la Educación. $\mathrm{N}^{\circ} 25$.

PRIGONINE, I. (1990). La nueva alianza Metamorfosis de la ciencia. Madrid: Alianza Editorial.

TEMPORETTI, F. (2008). Retos educativos del siglo XXI desde la perspectiva de América Latina Universidad Nacional de Rosario | (Argentina). Madrid. En http://www.fceia.unr.edu.ar/ geii/maestria/TEMPORETTI/Felix_Retos_Educativos_SXXI.

TEMPORETTI, F. (2008). Revolucionar la educación latinoamericana. Argentina: Universidad Nacional de Rosario.

TORRES, A. (2004). Por una investigación desde el margen. Colombia: CLACSO.

UNESCO (2005). Los problemas y desafíos de la educación en América Latina y el Caribe. Caracas.

VelÁSQueZ, A. y Rey, N. (1999). Metodología de la investigación científica. Lima: San Marcos. VEXLER, I. (2015). Educación para todos en América Latina y el Caribe. En http://www.cne. gob.pe/index.php/ldel-Vexler-Talledo/la-educacion-para-todos-en-america-latina-y-elcaribe.html 